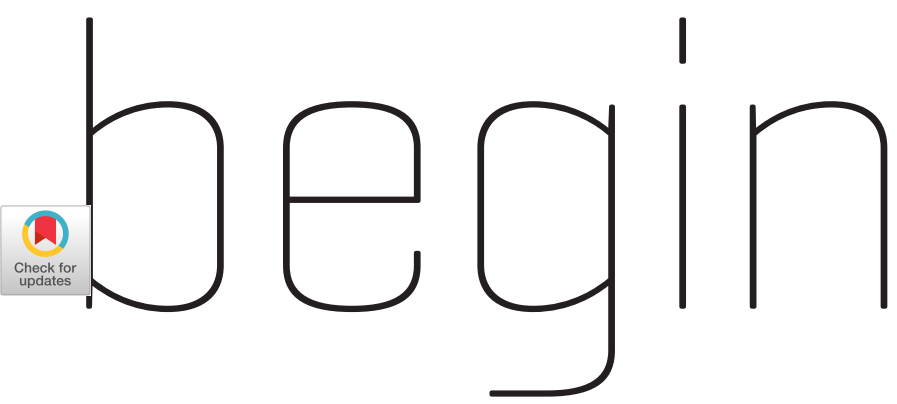

\section{Untangling Hype and Innovation}

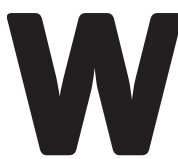

hen you start a new job, a new project, or any worthwhile new venture that involves risk, you often hear advice that goes along the lines of: "under-promise and over-deliver." At its core, this aphorism is about managing expectations and being successful at things that are tried and tested. When you underpromise and over-deliver, you force yourself to operate in a comfort zone where goals are achievable, uncertainty is minimized, and the stakeholders are happy. But what about the opposite- - over-promise and under-deliver"? Why would anyone align themselves to this philosophy when their money, their reputation, and their career could be at stake? Like it or not, the phenomenon of hyperbole happens frequently in the technology industry, where hype is deeply entrenched and normalized. If founders or innovators refuse to engage-either by painting a more toned-down version of their vision, or by being. skeptical about the latest and greatest - they run the risk of being painted as dispassionate, short sighted, or outright boring.

Even before Mary Shelley's "Frankenstein" novel published in 1818, the modern sci-fi genre has served as the mental sandbox in which the public could on one hand experiment with futuristic ideas, and on the other hand develop the psychological craving to turn these ideas into technological reality. From the telegraph [1] to the touch screen [2], most post-Enlightenment technological innovations were historically preceded by speculative stories in the print of their time. With each new innovation delivered, sensationalism increasingly established itself as the tool of choice for society to paint utopian visions, giving people something positive to hope for in the future, as well as the motivation to push themselves to overcome their condition.

We can therefore say that from the very beginning, hype has been a normal and natural cultural manifestation of technological progress and optimism.

Despite its normativity, however, hype also has a dark side. When taken too far, it turns into an incredibly destructive force: it creates unrealistic expectations, erodes trust, and causes irreparable financial and social harm. Case in point-tech bubbles.
The best known example of a tech bubble happened in the dot-com era, which started in 1997 and burst in 2001, bankrupting 50 percent of the Internet companies at the time and wiping out up to 80 percent of the stock value of most of the survivors [3].

Today, nowhere are bubbles more prevalent than in the volatile and reality-divorced culture of technology startups funded through venture capital, where inflationary hype led to the coining of terms such as "unicorn" and "super unicorn" [4]. These are companies with billions of dollars in valuation, despite having no proven track record of being profitable, or even producing anything tangible. It is for this reason that we chose the image of a unicorn on the cover to represent the essence of this Issue.

Our aim is to help youour student readers-to better understand hype and its cultural role, to develop a critical view of emerging technologies, and to be wary of unfounded promises of disruption and glory. Last but not least, we would like you to take away the lesson that lasting innovation is a function of time, hard work, and societal contexts. To illustrate

\section{In this issue, we bring you a wide range of perspectives on technological hype by specifically discussing why certain technologies fail while others succeed.}




When taken too
far, it [hype]
turns into an
incredibly
destructive
force: it creates
unrealistic
expectations,
erodes trust,
and causes
irreparable
financial and
social harm.

this point, we'll take you through two quick stories: one drawn from the past, and another that is fairly recent.

\section{STORY 1:}

THE PICTUREPHONE: A TECHNOLOGY 50 YEARS AHEAD OF ITS TIME

The Picturephone was first developed in 1956 by Bell Labs, the same institution who invented the transistor, radar, and information theory alongside other groundbreaking advancements. From the mid 1950 s to early 1970s, Bell Labs reportedly spent over $\$ 500$ million in $R \& D$, developing the picturephone system, on a mission to connect people through video. The predominant medium in that era was the telephone, and people thought that the next natural step would be video communication. Despite genuinely good intentions and huge financial backing by Bell Labs, picturephone adoption was extremely weak due to multiple factors. On one hand, the service was expensive (\$150/month at the time, the equivalent of about $\$ 1000$ in 2018 dollars). On the other hand, people realized that looking at someone while on the phone did not provide extra value beyond what a standard telephone can do. The high cost did not justify the value of the feature. Finally, having a dedicated device that could only do video conferencing. was not compelling enough to warrant adoption. By the 1970s, the picturephone had only about 100 subscribers in the entire USA, and by 1977, this number dropped to nine. Today, we have FaceTime [5].

\section{STORY 2:}

\section{THERANOS: FROM}

\$10 BILLION TO ZERO IN JUST ONE YEAR

Theranos was a private tech corporation founded in 2003 by Elizabeth Holmes on the claim that it could disrupt the healthcare industry by introducing revolutionary blood tests which only require a very small amount of blood from the patient. Owing in no small part to Holmes' strong social network, Theranos was able to quickly raise hundreds of millions of dollars in venture capital funding, with its valuation peaking at $\$ 10$ billion before crashing to basically zero by 2016. The downfall started after the Wall Street Journal published an article by John Carreyrou in October 2015 , in which it was exposed that the revolutionary technology Theranos had advertised did not, in fact, exist, and that all the blood tests were being run on traditional machines and were unreliable. The company came under criminal investigation for misleading investors and government officials about its technology, and it was formally dissolved in 2018, after Holmes and her partner were indicted on multiple counts of wire fraud [6].

\section{BEYOND HYPE}

In this issue, we bring you a wide range of perspectives on technological hype by specifically discussing why certain technologies fail while others succeed. As computer scientists, our excitement is heavily skewed towards novel, cool, and technologically "disruptive" ideas, but our editorial team argues that such a techno-centric viewpoint is often the cause of hype and failure. Socioeconomic and organizational factors play equally important roles in bringing innovation to fruition, but these factors are often deemed unsexy, and therefore get minimal attention. We invited several esteemed authors to discuss experiences from their respective fields, and our hope is that their insights will persuade you to take a more vigilant and holistic approach to innovation.

We begin with an incredibly remarkable story about the difficulties of commercializing a research idea. Dr. George Gerpheide, considered the father of the touchpad, takes you through his roller-coaster journey of bringing his research prototype to mass market-in his own words, an achievement that was anything but simple. Our hope is that his story will give you a better appre- 


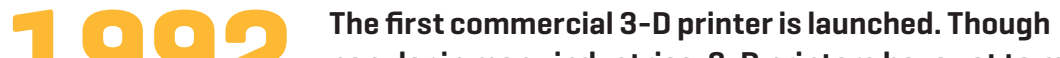 popular in many industries, 3-D printers have yet to make a successful entry into the homes of average consumers.}

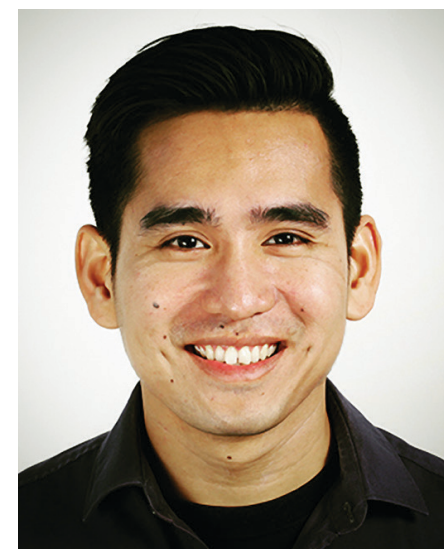

Gierad Laput

ciation for the amount of time and sustained effort required in building something that truly works as promised.

In addition to economic and productization constraints, innovation also requires a symbiotic interplay between technological and societal factors. MIT graduate student Amy X. Zhang discusses how the most successful technologies, old and new, are built on an intimate understanding of user dynamics. How do we scientifically explain, for example, that despite people clamoring for decades that email will die, we still use it? Amy's expertise is in the intersection of human-computer interaction and online communities, and her article discusses how technology inadvertently upends social norms in ways that can result in either delightful or disastrous outcomes.

Even if social constraints are successfully met, many

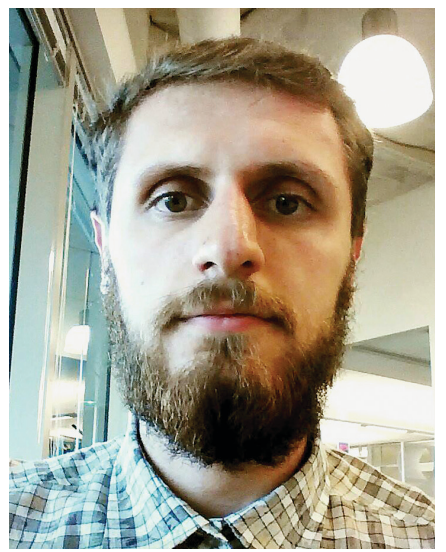

Adrian Scoica

technologies fail due to poor execution. This manifests itself through buggy software or products rife with usability issues. Bengt Sandblad and Thomas Lind discuss how these unmet user needs and blatant usability problems are catalysts to failure, and about how the problem is made worse in nonconsumer contexts such as hospitals. They also describe how these issues are often symptoms of a larger problem, one that stems from leadership and organizational inefficiencies.

As in the Picturephone story, the ability to identify a compelling use-case, colloquially referred to as the "killer application," is critical to technological success. We have three authors who discuss the disillusionment around previously hyped technologies, and they propose compelling ways forward for engaging with the subjects in question.
First, Siddharth Kumar Sah and Soumya Jindal discuss earlier hype in 3-D printing; they argue real innovation in this domain lies in bio printing instead.

Next, we turn our attention to one of the most recently hyped topics of our moment-artificial intelligence (AI). The term has been extremely overused and associated with such a dizzying array of technologies that it has almost lost its meaning. Moreover, AI hype comes from two different directions-one where AI is the all encompassing technology that can allegedly fix everything, and a dystopian view of AI where people lose jobs while robots take over the world. To help us navigate through this confusion, our esteemed feature editor, Johanna Schacht, synthesizes the most compelling and thought-provoking perspectives on what AI ought to be, and how we can move beyond the hype.

Finally, Jas Brooks, a graduate student at the University of Chicago, discusses the hype around virtual reality, and how applications beyond gaming, such as the museum experience, could become its most notable use-case.

We hope this issue plays an important role in equipping you with critical perspectives towards innovation. Fads and hype come and go (as has been happening throughout history), but true innovation is long lasting. We want you to be able to sift through the noise and to be skeptical about promises of the future. When we, computer scientists, better understand why hype happens and under what conditions it is beneficial, we can learn to become more grounded and truthful arbiters of the future we create.

- Gierad Laput
and Adrian Scoică,
Guest Editors

\section{References}

[1] C.M., Scots Magazine. February 17 Issue, 1753.

[2] Asimov, I. Foundation. Gnome Press, 1951.

[3] Berlin, L. Lessons of survival, from the dot-com attic. The New York Times, November 21, 2008.

[4] Lee, A. Welcome To the unicorn club: learning from billion-dollar startups. TechCrunch, 2013.

[5] Gertner, J. The Idea Factory: Bell Labs and the Great Age of American Innovation. Penguin Books, New York, 2013.

[6] Carreyrou, J. Bad blood: Secrets and Lies in a Silicon Valley Startup. Alfred A. Knopf, New York, 2018.

\section{Biographies}

Gierad Laput is a Ph.D. candidate at the School of Computer Science at Carnegie Mellon University. His research in human-computer interaction designs, builds, and evaluates novel interactive technologies that greatly enhances input expressivity for users and contextual awareness for devices. His research lies at the intersection of interactive systems, sensing, and applied machine learning.

Adrian Scoică is a computer engineer and scientist currently working on fake account detection at Google in Switzerland. Prior to fake account detection, he was working on Natural Language Processing at the University of Cambridge [UK]

DOI: $10.1145 / 3302252$ Copyright held by authors. 